\title{
Applications of the Kelmans transformation: extremality of the threshold graphs
}

\author{
Péter Csikvári* \\ Eötvös Loránd University, Department of Computer Science \\ H-1117 Budapest Pázmány Péter sétány 1/C, Hungary \\ Alfréd Rényi Institute of Mathematics \\ H-1053 Budapest Reáltanoda u. 13-15, Hungary \\ csiki@cs.elte.hu
}

Submitted: June 16, 2011; Accepted: Sep 7, 2011; Published: Sep 20, 2011

Mathematics Subject Classifications: 05C31

\begin{abstract}
In this paper we study various extremal problems related to some combinatorially defined graph polynomials such as matching polynomial, chromatic polynomial, Laplacian polynomial. It will turn out that many problems attain its extremal value in the class of threshold graphs. To attack these kinds of problems we survey several applications of the so-called Kelmans transformation.
\end{abstract}

\section{Introduction}

Let $N(x)$ denote the set of neighbors of the vertex $x$. Then the threshold graphs are most easily defined as those graphs for which every vertices $u$ and $v$, the sets $N(u) \backslash\{v\}$ and $N(v) \backslash\{u\}$ are comparable respected to set-inclusion.

In this paper we show that various extremal problems on combinatorially defined polynomials of graphs have its maximum or minimum attained at a threshold graph. Our results will have the following shape: let $P_{G}(x)=x^{n}+a_{n-1} x^{n-1}+\cdots+a_{0}$ be some polynomial of the graph $G$ (for example matching polynomial, chromatic polynomial) then there exist a degree-maximal graph $G^{*}$ with the same number of edges such that for the polynomial $P_{G^{*}}(x)=x^{n}+b_{n-1} x^{n-1}+\cdots+b_{0}$ we have $\left|a_{k}\right| \leq\left|b_{k}\right|$ (or $\left|a_{k}\right| \geq\left|b_{k}\right|$ ) for all $0 \leq k \leq n-1$ or the largest (smallest) real root of the polynomial $P_{G}$ is greater (smaller) than that of $P_{G^{*}}$; the exact relation depends on the type of polynomial (e. g., for the

\footnotetext{
${ }^{*}$ The research was partially supported by the Hungarian National Foundation for Scientific Research (OTKA), Grant no. K 81310
} 
matching polynomial we show that $\left|b_{k}\right| \leq\left|a_{k}\right|$ while for the independence polynomial we will show that $\left|b_{k}\right| \geq\left|a_{k}\right|$ for all $\left.0 \leq k \leq n-1\right)$. We will distinguish this two type of results as coefficient majorization result and root majorization result.

Our main tool will be the so-called Kelmans-transformation. This transformation controls efficiently many graph parameters and the threshold graphs of this transformation are exactly the graphs known as threshold graphs.

The rest of the paper is organized as follows. In Section 2 we introduce the concept of the Kelmans transformation. In Section 3 we give a coefficient majorization result for the matching polynomial as a warm-up. In Section 4 we prove a root majorization result for the matching polynomial. In Section 5 we present a coefficient majorization and a root majorization result for the independence polynomial, while in Section 6 we give a coefficient majorization result for the chromatic polynomial. In Section 7 we prove a lemma on the effect of the Kelmans transformation on the so-called exponential-type graph polynomials. Using this lemma we prove a coefficient majorization result for the Laplacian polynomial in Section 8. In Section 9 we give an application of the so-called NA-Kelmans transformation on the number of closed walks. In Section 10 we give the studied graph polynomials of the threshold graphs. We end the paper with some remarks on the use of the Kelmans transformation.

We note that some of the above mentioned results are very easy, but others requires tedious preparations. In fact, the root majorization results and the coefficient majorization result of the Laplacian polynomial can be considered as the main results of this paper.

Notation: Throughout the paper we will consider only simple graphs. We will follow the usual notation: $G$ is a graph, $V(G)$ is the set of its vertices, $E(G)$ is the set of its edges, $e(G)$ denotes the number of edges, $N(x)$ is the set of the neighbors of $x$, $\left|N\left(v_{i}\right)\right|=\operatorname{deg}\left(v_{i}\right)=d_{i}$ denote the degree of the vertex $v_{i}$. We will also use the notation $N[v]$ for the closed neighbor $N(v) \cup\{v\}$.

For $S \subset V(G)$ the graph $G-S$ denotes the subgraph of $G$ induced by the vertices $V(G) \backslash S$ while $\left.G\right|_{S}$ denotes the subgraph of $G$ induced by the vertex set $S$. If $e \in E(G)$ then $G-e$ denotes the graph with vertex set $V(G)$ and edge set $E(G) \backslash\{e\}$. We also use the notation $G / e$ for the graph obtained from $G$ by contracting the edge $e$; clearly the resulting graph is multigraph.

For polynomials $P_{1}$ and $P_{2}$ we will write $P_{1}(x) \gg P_{2}(x)$ if they have the same degree and the absolute value of the coefficient of $x^{k}$ in $P_{1}(x)$ is at least as large as the absolute value of the coefficient of $x^{k}$ in $P_{2}(x)$ for all $0 \leq k \leq n$.

Additional definitions and notation will be given in the sections.

\section{Kelmans transformation}

In [13] Kelmans studied the following problem. Let $R_{q}^{k}(G)$ be the probability that if we remove the edges of the graph $G$ with probability $q$, independently of each other, then the obtained random graph has at most $k$ components. He obtained many results on extremal 
values of the parameter $R_{q}^{k}($.$) and on comparing graphs according to this parameter. One$ of his results was that a certain transformation increases this probability for every $q$. The study of this transformation (or more precisely its inverse), which we will call Kelmans transformation, will be the main tool in this paper.

Definition 2.1. Let $u, v$ be two vertices of the graph $G$, we obtain the Kelmans transformation of $G$ as follows: we erase all edges between $v$ and $N(v) \backslash(N(u) \cup\{u\})$ and add all edges between $u$ and $N(v) \backslash(N(u) \cup\{u\})$. Let us call $u$ and $v$ the beneficiary and the co-beneficiary of the transformation, respectively. The obtained graph has the same number of edges as $G$; in general we will denote it by $G^{\prime}$ without referring to the vertices $u$ and $v$.
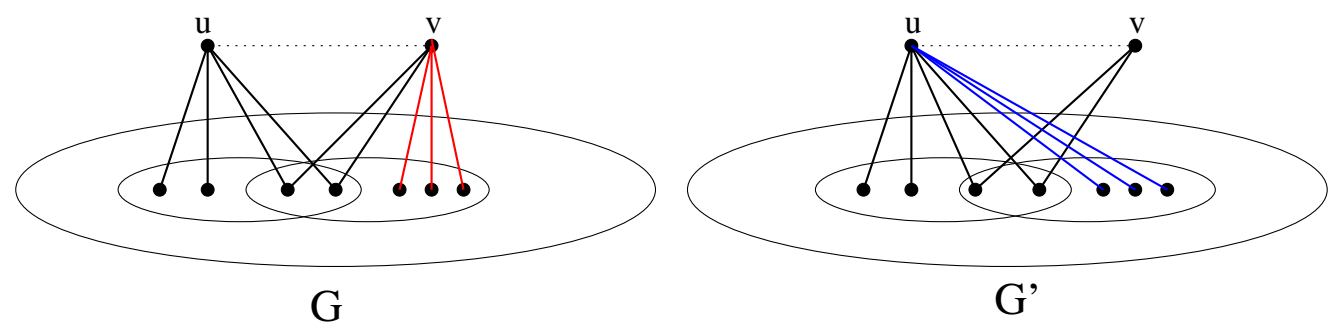

Figure 1: The Kelmans transformation.

The original application of the Kelmans transformation was the following (see Theorem 3.2 of [13]). We note that we use our notation.

Theorem 2.2. [13] Let $G$ be a graph and $G^{\prime}$ be a graph obtained from $G$ by a Kelmans transformation. Then $R_{q}^{k}(G) \geq R_{q}^{k}\left(G^{\prime}\right)$ for every $q \in(0,1)$.

Satyanarayana, Schoppmann and Suffel [19] rediscovered Theorem 2.2, they called the inverse of the Kelmans transformation "swing surgery". They also proved the following theorem which we will also use and prove.

Theorem 8.5. [19] Let $G$ be a graph and $G^{\prime}$ be a graph obtained from $G$ by a Kelmans transformation. Let $\tau(G)$ and $\tau\left(G^{\prime}\right)$ be the number of spanning trees of the graph $G$ and $G^{\prime}$, respectively. Then $\tau\left(G^{\prime}\right) \leq \tau(G)$.

Brown, Colbourn and Devitt [3] studied the Kelmans transformation further in the context of network reliability. They also extended it to multigraphs. We will primarily concern with simple graphs, but we show that the Kelmans transformation can be applied efficiently in a much wider range of problems. In [5] the author proved the following result concerning the spectral radius of the adjacency matrix.

Theorem 2.3. [5] Let $\mu(H)$ denote the largest eigenvalue of the adjacency matrix of the graph $H$. Let $G$ be a graph and let $G^{\prime}$ be a graph obtained from $G$ by some Kelmans transformation. Then

$$
\mu\left(G^{\prime}\right) \geq \mu(G)
$$


Remark 2.4. The $\{u, v\}$-independence and the Nordhaus-Gaddum property of the Kelmans transformation. The key observation is that up to isomorphism $G^{\prime}$ is independent of $u$ or $v$ being the beneficiary or the co-beneficiary if we apply the transformation to $u$ and $v$. Indeed, in $G^{\prime}$ one of $u$ or $v$ will be adjacent to $N_{G}(u) \cup N_{G}(v)$, the other will be adjacent to $N_{G}(u) \cap N_{G}(v)$ (and if the two vertices are adjacent in $G$ then they will remain adjacent, too). This observation also implies that the Kelmans transformation is also a Kelmans transformation to the complement of the graph $G$ with the change of the role of $u$ and $v$.

This means that whenever we prove that the Kelmans transformation increases some parameter $p(G)$, i.e., $p\left(G^{\prime}\right) \geq p(G)$ then we immediately obtain that $p\left(\overline{G^{\prime}}\right) \geq p(\bar{G})$ as well. This observation is particularly fruitful in those problems where one considers a graph and its complement together like in Nosal's problem of bounding the sum of the spectral radii of the graph $G$ and its complement. The following result of this type was obtained in $[5]$.

Theorem 2.5. [5]

$$
\begin{gathered}
\mu(G)+\mu(\bar{G}) \leq \frac{1+\sqrt{3}}{2} n . \\
\star \star \star
\end{gathered}
$$

We end this section by some remarks on the threshold graphs of this transformation. We show that the threshold graphs of the Kelmans transformation are exactly the graphs known as threshold graphs.

Let us say that $u$ dominates $v$ if $N(v) \backslash\{u\} \subseteq N(u) \backslash\{v\}$. Clearly, if we apply the Kelmans transformation to a graph $G$ and $u$ and $v$ such that $u$ is the beneficiary then $u$ will dominate $v$ in $G^{\prime}$. If neither $u$ dominates $v$, nor $v$ dominates $u$ we say that $u$ and $v$ are incomparable; in this case we call the Kelmans transformation applied to $u$ and $v$ proper. One can prove the following simple statement. (The proof of part (a) of this theorem can be found in [5].)

Theorem 2.6. (a) By the application of a sequence of Kelmans transformation one can always transform an arbitrary graph $G$ to a graph $G_{t r}$ in which the vertices can be ordered so that whenever $i<j$ then $v_{i}$ dominates $v_{j}$.

(b) Furthermore, one can assume that $G_{t r}$ has exactly the same number of components as $G$. (Note that all but one component of a threshold graph $G_{t r}$ are isolated vertices.)

We also mention the following very simple statement.

Theorem 2.7. [15] A graph $G$ is the threshold graph of the Kelmans transformation if and only if it can be obtained from the empty graph by the following steps: adding some isolated vertices to the graph or complementing the graph.

Remark 2.8. Note that the graphs described in the previous theorem are called "threshold graphs" in the literature. Hence the threshold graphs of the Kelmans transformation are exactly the threshold graphs. (It seems to me that this statement is nontrivial in the 


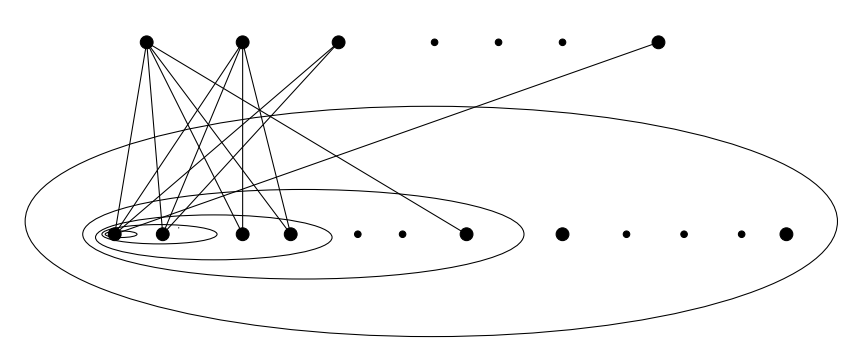

Figure 2: A threshold graph of the Kelmans transformation.

sense that the threshold graphs are called threshold graphs not because of the Kelmans transformation.) From now on we simply refer to these graphs as threshold graphs.

Remark 2.9. These graphs, or more precisely their adjacency matrices also appear in the article of Brualdi and Hoffman [4]. Rowlinson called these matrices stepwise matrices [18].

\section{The number of matchings}

In this section we study the matching polynomials of graphs. For fundamental results on matching polynomials see $[9,11,12]$.

Definition 3.1. Let $m_{r}(G)$ denote the number of $r$ independent edges (,i.e., the $r$ matchings) in the graph $G$. Define the matching polynomial of $G$ as

$$
\mu(G, x)=\sum_{r=0}(-1)^{r} m_{r}(G) x^{n-2 r} .
$$

Theorem 3.2. Assume that $G^{\prime}$ is a graph obtained from $G$ by some Kelmans transformation, then

$$
\mu(G, x) \gg \mu\left(G^{\prime}, x\right) .
$$

In other words, $m_{r}(G) \geq m_{r}\left(G^{\prime}\right)$ for $1 \leq r \leq n / 2$. In particular, the Kelmans transformation decreases the maximum number of independent edges.

Remark 3.3. I invite the Reader to prove this theorem on their own; although I give the proof of the theorem here, it takes much longer to read it then to prove it on their own.

Proof. We need to prove that for every $r$ the Kelmans transformation decreases the number of $r$-matchings. Assume that we applied the Kelmans transformation to $G$ such that $u$ was the beneficiary and $v$ was the co-beneficiary. Furthermore, let $\mathcal{M}_{r}(G)$ and $\mathcal{M}_{r}\left(G^{\prime}\right)$ denote the set of $r$-matchings in $G$ and $G^{\prime}$, respectively. We will give an injective map from $\mathcal{M}_{r}\left(G^{\prime}\right)$ to $\mathcal{M}_{r}(G)$.

In those cases where all edges of the $r$-matching of $G^{\prime}$ are also edges in $G$ we simply take the identical map. 
Next consider those cases where $v$ is not covered by the matching, but for some $w \in$ $N_{G}(v) \backslash N_{G}(u)$ we have $u w$ in the $r$-matching. Map this $r$-matching to the $r$-matching obtained by exchanging $u w$ to $v w$ in the $r$-matching, but otherwise we do not change the other edges of the matching. Clearly, the image will be an $r$-matching of $G$ and since $v w \notin E\left(G^{\prime}\right)$ this is not in the image of the previous case.

Finally, consider those cases where both $u$ and $v$ are covered in the $r$-matching of $G^{\prime}$ and the $r$-matching does not belong to the first case. In this case there exist a $w_{1} \in$ $N_{G}(v) \backslash N_{G}(u)$ and a $w_{2} \in N_{G}(v) \cap N_{G}(u)$ such that $u w_{1}$ and $v w_{2}$ are in the $r$-matching of $G^{\prime}$. Let the image of this $r$-matching be defined as follows. We exchange $u w_{1}$ and $v w_{2}$ to $u w_{2}$ and $v w_{1}$ in $G$, but otherwise we leave the other $r-2$ edges of the $r$-matching. Clearly we get an $r$-matching of $G$ and the image of this $r$-matching is not in the image of the previous cases, because both $u$ and $v$ are covered (not as in the second case) and $v w_{1} \in E(G)$ is in the $r$-matching (not as in the first case).

Hence we have given an injective map from $\mathcal{M}_{r}\left(G^{\prime}\right)$ to $\mathcal{M}_{r}(G)$ proving that $m_{r}\left(G^{\prime}\right) \leq$ $m_{r}(G)$.

We mentioned that the Kelmans transformation is also Kelmans transformation of the complement of the graph. As an example one can prove the following (very simple) result on maximal matchings. We left the details to the Reader.

Corollary 3.4. Let $G$ be a graph on $n$ vertices. Then $G$ or $\bar{G}$ contains $\left\lfloor\frac{n}{3}\right\rfloor$ independent edges.

Remark 3.5. The statement is best possible as it is shown by the clique of size $\frac{2 n}{3}$ and additional $\frac{n}{3}$ isolated vertices.

Corollary 3.4 is well-known, in fact, it is a motivating result of several colored matching problem, see e.g. [6].

\section{The largest root of the matching polynomial}

It is a well-known theorem of Heilmann and Lieb [12] that all the roots of the matching polynomial are reals; so it is meaningful to speak about its largest root. In this section we will show that the Kelmans transformation increases the largest root of the matching polynomial (see Theorem 4.4). To do this we need some preparation.

Definition 4.1. Let $t(G)$ be the largest root of the matching polynomial $\mu(G, x)$. Furthermore let $G_{1} \succ G_{2}$ if for all $x \geq t\left(G_{1}\right)$ we have $\mu\left(G_{2}, x\right) \geq \mu\left(G_{1}, x\right)$.

Proposition 4.2. The relation $\succ$ is transitive and if $G_{1} \succ G_{2}$ then $t\left(G_{1}\right) \geq t\left(G_{2}\right)$.

Proof. Let $G_{1} \succ G_{2}$. Since $\mu\left(G_{1}, x\right)$ has positive leading coefficient and $t\left(G_{1}\right)$ is the largest root we have $\mu\left(G_{1}, x\right)>0$ for $x>t\left(G_{1}\right)$. Since $\mu\left(G_{2}, x\right) \geq \mu\left(G_{1}, x\right)>0$ on the interval $\left(t\left(G_{1}\right), \infty\right)$ we have $t\left(G_{2}\right) \leq t\left(G_{1}\right)$. If $G_{1} \succ G_{2} \succ G_{3}$ then $\mu\left(G_{3}, x\right) \geq \mu\left(G_{2}, x\right) \geq \mu\left(G_{1}, x\right)$ on the interval $\left[\max \left(t\left(G_{2}\right), t\left(G_{1}\right)\right), \infty\right)=\left[t\left(G_{1}\right), \infty\right)$, i.e., $G_{1} \succ G_{3}$. 
We will use the following two facts about the matching polynomial. The first one is the well-known recursion formula for the matching polynomials. The second fact is a result of D. Fisher and J. Ryan [8], it was a corollary of their theorem on the dependence polynomials; in Section 5 we will give an alternative proof of this result, see Corollary 5.7.

Fact 1. $[9,11,12]$ Let $e=u v \in E(G)$. Then we have the following recursion formula for matching polynomials

$$
\mu(G, x)=\mu(G-e, x)-\mu(G \backslash\{u, v\}, x) .
$$

Fact 2. [8] If $G_{2}$ is a subgraph of $G_{1}$ then $t\left(G_{1}\right) \geq t\left(G_{2}\right)$.

Proposition 4.3. If $G_{2}$ is a spanning subgraph of $G_{1}$ then $G_{1} \succ G_{2}$.

Proof. By the transitivity of the relation $\succ$ it is enough to prove the statement when $G_{2}=G_{1}-e$ for some edge $e=u v$. By Fact 1 . we have

$$
\mu(G, x)=\mu(G-e, x)-\mu(G \backslash\{u, v\}, x) .
$$

Since $G \backslash\{u, v\}$ is a subgraph of $G$ we have $t(G \backslash\{u, v\}) \leq t(G)$ by Fact 2. Since the main coefficient of $\mu(G \backslash\{u, v\})$ is 1 , this implies that for $x \geq t(G)$ we have $\mu(G \backslash\{u, v\}, x) \geq 0$. By the above identity we get $G \succ G-e$.

Theorem 4.4. Assume that $G^{\prime}$ is a graph obtained from $G$ by some Kelmans transformation, then $G^{\prime} \succ G$, in particular $t\left(G^{\prime}\right) \geq t(G)$.

Proof. Let $u, v$ be the two vertices of the graph $G$ for which we apply the Kelmans transformation such that $u$ is the beneficiary. We will prove that $G^{\prime} \succ G$; according to Proposition 4.2 this implies that $t\left(G^{\prime}\right) \geq t(G)$. We will prove this claim by induction on the number of edges of $G$.

Let us choose a vertex $w$ different from $v$ such that $u w \in E(G)$. If such $w$ does not exist then $G^{\prime}$ is isomorphic to $G$ and the claim is trivial. Thus we can assume that such a $w$ exists, let $h=u w$. Now we can write up the identities of Fact 1:

$$
\mu(G, x)=\mu(G-h, x)-\mu(G-\{u, w\}, x)
$$

and

$$
\mu\left(G^{\prime}, x\right)=\mu\left(G^{\prime}-h, x\right)-\mu\left(G^{\prime}-\{u, w\}, x\right) .
$$

Here $G^{\prime}-h$ can be obtained from $G-h$ by some Kelmans transformation and these graphs have less number of edges than $G$; so by induction we have $G^{\prime}-h \succ G-h$, i.e.,

$$
\mu(G-h, x) \geq \mu\left(G^{\prime}-h, x\right)
$$


for all $x \geq t\left(G^{\prime}-h\right)$. On the other hand $G^{\prime}-\{u, w\}$ is a spanning subgraph of $G-\{u, w\}$, thus we have $G-\{u, w\} \succ G^{\prime}-\{u, w\}$ by Proposition 4.3. In other words,

$$
\mu\left(G^{\prime}-\{u, w\}, x\right) \geq \mu(G-\{u, w\}, x)
$$

for all $x \geq t(G-\{u, w\})$. Altogether we get that

$$
\begin{gathered}
\mu(G, x)=\mu(G-h, x)-\mu(G-\{u, w\}, x) \geq \\
\geq \mu\left(G^{\prime}-h, x\right)-\mu\left(G^{\prime}-\{u, w\}, x\right)=\mu\left(G^{\prime}, x\right)
\end{gathered}
$$

for all $x \geq \max \left(t\left(G^{\prime}-h\right), t(G-\{u, w\})\right)$. Note that $t\left(G^{\prime}\right) \geq \max \left(t\left(G^{\prime}-e\right), t(G-\{u, w\})\right)$ as both graphs are subgraphs of $G$ (so we can use Fact 2); in the latter case we embed the graph $G-\{u, w\}$ into $G^{\prime}$ such that $v$ goes to $u$ in the embedding. Thus

$$
\mu(G, x) \geq \mu\left(G^{\prime}, x\right)
$$

for all $x \geq t\left(G^{\prime}\right)$.

Hence $G^{\prime} \succ G$ and we have proved the theorem.

\section{The independence polynomial}

We define the independence polynomial as follows.

Definition 5.1. Let $i_{k}(G)$ denote the number of independent sets of size $k$. Then we define the independence polynomial of the graph $G$ as

$$
I(G, x)=\sum_{k=0}^{n}(-1)^{k} i_{k}(G) x^{k} .
$$

Let $\beta(G)$ denote the smallest real root of $I(G, x)$; it exists and it is positive by the alternating sign of the coefficients of the polynomial.

Remark 5.2. Some authors call the polynomial $I(G,-x)$ the independence polynomial; since the transformation between the two forms is trivial it will not cause any confusion to work with this definition.

The graph parameter $\beta(G)$ is examined in various papers. D. Fisher and J. Ryan [8] proved that the (in)dependence polynomial always has a real root having the smallest absolute value among the roots. They also proved the following fundamental result on $\beta(G)$ : if $G_{1}$ is a subgraph of $G_{2}$ then $\beta\left(G_{1}\right) \geq \beta\left(G_{2}\right)$.

In this section we prove that the Kelmans transformation decreases the smallest real root of the independence polynomial.

We will use the following recursion formulas of the independence polynomials subsequently. 
Fact 1. ([14]) The polynomial $I(G, x)$ satisfies the recursion

$$
I(G, x)=I(G-v, x)-x I(G-N[v], x),
$$

where $v$ is an arbitrary vertex of the graph $G$.

Fact 2. ([14]) The polynomial $I(G, x)$ satisfies the recursion

$$
I(G, x)=I(G-e, x)-x^{2} I(G-N[v]-N[u], x),
$$

where $e=(u, v)$ is an arbitrary edge of the graph $G$.

We are going to prove our result in an analogous way that we have seen at the matching polynomials.

Definition 5.3. Let $G_{1} \succ G_{2}$ if $I\left(G_{2}, x\right) \geq I\left(G_{1}, x\right)$ on the interval $\left[0, \beta\left(G_{1}\right)\right]$.

This definition seems to be unnatural, because of the "reversed" inequality, but one can prove that if $G_{2}$ is a subgraph of $G_{1}$ then $G_{1} \succ G_{2}$ (see Proposition 5.6). Thus in the light of the following statement this claim implies Fisher and Ryan's result (see Remark 5.2).

Proposition 5.4. The relation $\succ$ is transitive on the set of graphs and if $G_{1} \succ G_{2}$ then $\beta\left(G_{1}\right) \leq \beta\left(G_{2}\right)$.

Proof. Let $G_{1} \succ G_{2}$. Since $I\left(G_{1}, 0\right)=1$ we have $I\left(G_{1}, x\right)>0$ on the interval $\left[0, \beta\left(G_{1}\right)\right)$. Thus $I\left(G_{2}, x\right) \geq I\left(G_{1}, x\right)>0$ on the interval $\left[0, \beta\left(G_{1}\right)\right)$ giving that $\beta\left(G_{2}\right) \geq \beta\left(G_{1}\right)$. If $G_{1} \succ G_{2} \succ G_{3}$ then $\beta\left(G_{1}\right) \leq \beta\left(G_{2}\right) \leq \beta\left(G_{3}\right)$ and $I\left(G_{3}, x\right) \geq I\left(G_{2}, x\right) \geq I\left(G_{1}, x\right)$ on the interval $\left[0, \min \left(\beta\left(G_{1}\right), \beta\left(G_{2}\right)\right)\right)=\left[0, \beta\left(G_{1}\right)\right)$ thus $G_{1} \succ G_{3}$.

Proposition 5.5. If $G_{2}$ is an induced subgraph of $G_{1}$ then $G_{1} \succ G_{2}$.

Proof. We prove by induction on the number of vertices of $G_{1}$. For sake of simplicity let us use the notation $G_{1}=G$. By the transitivity of the relation $\succ$ it is enough to prove that $G \succ G-v$. The statement is true if $|V(G)|=2$.

Since $G-N[v]$ is an induced subgraph of $G-v$, by the induction hypothesis we have

$$
I(G-v, x) \succ I(G-N[v], x) .
$$

This means that

$$
I(G-N[v], x) \geq I(G-v, x)
$$

on the interval $[0, \beta(G-v)]$. Thus $I(G-N[v], x) \geq 0$ on the interval $[0, \beta(G-v)]$. Hence by Fact 1 we have $I(G, x) \leq I(G-v, x)$ on the interval $[0, \beta(G-v)]$. This implies that $\beta(G) \leq \beta(G-v) ; I(G, 0)=1$ and $I(G, \beta(G-v)) \leq 0$ so $I(G, x)$ has a root in the interval $[0, \beta(G-v)]$. Hence $I(G, x) \leq I(G-v, x)$ on the interval $[0, \beta(G)]$, i.e., $G \succ G-v$. 
Proposition 5.6. If $G_{2}$ is a subgraph of $G_{1}$ then $G_{1} \succ G_{2}$.

Proof. Let us apply the notation $G_{1}=G$.

Clearly, it is enough to prove that $G \succ G-e$ where $e=(u, v) \in E(G)$. Let us use the recursion formula of Fact 2 to $G$ :

$$
I(G, x)=I(G-e, x)-x^{2} I(G-N[u]-N[v], x) .
$$

By Proposition 5.5 we have $G \succ G-N[u]-N[v]$ and so

$$
I(G-N[u]-N[v], x) \geq I(G, x) \geq 0
$$

on the interval $[0, \beta(G)]$. Hence $I(G-e, x) \geq I(G, x)$ on this interval, i.e. , $G \succ G-e$.

Corollary 5.7. If $G_{1}$ is a subgraph of $G_{2}$ then $t\left(G_{1}\right) \leq t\left(G_{2}\right)$ where $t\left(G_{1}\right)$ and $t\left(G_{2}\right)$ are the largest roots of the matching polynomial of $G_{1}$ and $G_{2}$, respectively.

Proof. One can transform the matching polynomial into the independence polynomial of the line graph.

The main result of this section is the following

Theorem 5.8. The Kelmans transformation decreases the smallest root of the independence polynomial. More precisely, assume that $G^{\prime}$ is a graph obtained from $G$ by some Kelmans transformation, then $G^{\prime} \succ G$ and so $\beta\left(G^{\prime}\right) \leq \beta(G)$.

Proof. We prove the statement by induction on the number of vertices. The claim is true for small graphs. Let $u$ be the beneficiary at the Kelmans transformation, $v$ be the co-beneficiary. We can assume that $N_{G}(u) \backslash N_{G}(v)$ is not empty, otherwise $G^{\prime}$ and $G$ are isomorphic, so let $w \in N_{G}(u) \backslash N_{G}(v)$. Now let us use the recursion formula of Fact 1

$$
I(G, x)=I(G-w, x)-x I\left(G-N_{G}[w], x\right)
$$

and

$$
I\left(G^{\prime}, x\right)=I\left(G^{\prime}-w, x\right)-x I\left(G^{\prime}-N_{G^{\prime}}[w], x\right) .
$$

Observe that $G^{\prime}-w$ can be obtained from $G-w$ by some Kelmans transformation and so by the induction we have

$$
I(G-w, x) \geq I\left(G^{\prime}-w, x\right)
$$

on the interval $\left[0, \beta\left(G^{\prime}-w\right)\right]$. On the other hand, $G^{\prime}-N_{G^{\prime}}[w]$ is a subgraph of $G-N_{G}[w]$, thus by Proposition 5.6 we have

$$
I\left(G^{\prime}-N_{G^{\prime}}[w], x\right) \geq I\left(G-N_{G}[w], x\right)
$$

on the interval $\left[0, \beta\left(G-N_{G}[w]\right)\right]$. Putting together these two inequalities we get that

$$
I(G, x) \geq I\left(G^{\prime}, x\right)
$$

on the interval $\left[0, \min \left(\beta\left(G^{\prime}-w\right), \beta\left(G-N_{G}[w]\right)\right]\right.$. Note that $G^{\prime}-w$ and $G-N_{G}[w]$ are both subgraphs of $G^{\prime}$; in the latter case $v$ goes to $u$ at the injective homomorphism from $V\left(G-N_{G}[w]\right)$ to $V\left(G^{\prime}\right)$. Thus we have $\beta\left(G^{\prime}\right) \leq \min \left(\beta\left(G^{\prime}-w\right), \beta\left(G-N_{G}[w]\right)\right)$. This proves that $G^{\prime} \succ G$. 
Remark 5.9. Theorem 5.8 does not imply Theorem 4.4 since the Kelmans transformation on a graph $G$ does not induce a Kelmans transformation on the line graph.

\subsection{The number of independent sets}

Theorem 5.10. The Kelmans transformation increases the number of independent sets of size $r$ and the number of cliques of size $r$, i.e., assume that $G^{\prime}$ is a graph obtained from $G$ by some Kelmans transformation, then $i_{r}(G) \leq i_{r}\left(G^{\prime}\right)$ and $i_{r}(\bar{G}) \leq i_{r}\left(\overline{G^{\prime}}\right)$ for all $r$.

Disclaimer: it is easier to prove this theorem on their own than to read the following proof.

Proof. Since the Kelmans transformation of the graph $G$ is also a Kelmans transformation of its complement, it is enough to prove the statement concerning the number of cliques of size $k$. Let $\mathcal{C} l_{k}(G)$ and $\mathcal{C} l_{k}\left(G^{\prime}\right)$ be the set of cliques of size $k$ in $G$ and $G^{\prime}$, respectively. We will give an injective map $\varphi$ from $\mathcal{C} l_{k}(G)$ to $\mathcal{C} l_{k}\left(G^{\prime}\right)$. This way we prove that $\left|\mathcal{C} l_{k}(G)\right| \leq$ $\left|\mathcal{C} l_{k}\left(G^{\prime}\right)\right|$.

Let $S \in \mathcal{C} l_{k}(G)$. If $S \in \mathcal{C} l_{k}\left(G^{\prime}\right)$ then we simply define $\varphi$ to be the identity map. If $S \notin \mathcal{C} l_{k}\left(G^{\prime}\right)$ then $v \in V(S)$ and there exists some $w \in N_{G}(v) \backslash N_{G}(u)$ for which $w \in V(S)$ as well. This implies that $u \notin V(S)$. In this case let $\varphi(S)$ be the clique of $G^{\prime}$ induced on the set $(S-v) \cup\{u\}$. This is indeed a clique of $G^{\prime}$ and it cannot be the clique of $G$ so it is not the image of any other clique of $G$. Hence $\varphi$ is injective.

\section{The chromatic polynomial}

In this section we prove a coefficient majorization result for the chromatic polynomial, see Theorem 6.3 below.

Recall that we define the chromatic polynomial $\operatorname{ch}(G, \lambda)$ of the graph $G$ as follows $[2,17]$ : for a positive integer $\lambda$ the value $\operatorname{ch}(G, \lambda)$ is the number of ways that $G$ can be well-colored with $\lambda$ colors. It is indeed a polynomial in $\lambda$ :

$$
\operatorname{ch}(G, \lambda)=\sum_{k=1}^{n}(-1)^{n-k} c_{k}(G) \lambda^{k}
$$

The coefficients of the chromatic polynomial have the following nice interpretation [2].

Theorem 6.1. Let $G$ be a graph on $n$ vertices and edge set $E(G)=\left\{e_{1}, e_{2}, \ldots, e_{m}\right\}$. Call a subset of $E(G)$ a broken cycle if it is obtained from the edge set of a cycle by deleting the edge of highest index. Then the chromatic polynomial of $G$ is

$$
\operatorname{ch}(G, \lambda)=\lambda^{n}-c_{n-1} \lambda^{n-1}+c_{n-2} \lambda^{n-2}-\cdots+(-1)^{n-1} c_{1} \lambda,
$$

where $c_{i}$ is the number of $n-i$-subsets of $E(G)$ containing no broken cycles. 
Remark 6.2. In fact, we will only need that the coefficients of the chromatic polynomial have alternating sign. This can easily be deduced from the recursion formula of Proposition 6.4 too.

Theorem 6.3. The Kelmans transformation decreases the coefficients of the chromatic polynomial in absolute value, i.e., assume that $G^{\prime}$ is a graph obtained from $G$ by some Kelmans transformation, then

$$
\operatorname{ch}(G, \lambda) \gg \operatorname{ch}\left(G^{\prime}, \lambda\right) .
$$

In other words, $c_{k}(G) \geq c_{k}\left(G^{\prime}\right)$ for $k=1, \ldots, n-1$.

To prove this theorem we need some preparation.

Proposition 6.4. $[2,17]$ Let $e \in E(G)$ then

$$
\operatorname{ch}(G, \lambda)=\operatorname{ch}(G-e, \lambda)-\operatorname{ch}(G / e, \lambda) .
$$

Lemma 6.5. If $G_{1}$ is a spanning subgraph of $G$ then

$$
\operatorname{ch}(G, \lambda) \gg \operatorname{ch}\left(G_{1}, \lambda\right) .
$$

Proof. It is enough to prove the claim for $G_{1}=G-e$ for which the statement is trivial by Proposition 6.4 and Theorem 6.1.

Now we are ready to prove Theorem 6.3.

Proof. Let us introduce the notation

$$
\widehat{c h}(G, \lambda)=(-1)^{|V(G)|} \operatorname{ch}(G,-\lambda) .
$$

Then $\widehat{c h}(G, \lambda)=\sum_{k=1}^{n} c_{k}(G) \lambda^{k}$ has only non-negative coefficients. Clearly, one can rewrite Proposition 6.4 as

$$
\widehat{c h}(G, \lambda)=\widehat{c h}(G-e, \lambda)+\widehat{c h}(G / e, \lambda) .
$$

We need to prove that $\widehat{c h}(G, \lambda) \gg \widehat{c h}\left(G^{\prime}, \lambda\right)$.

We prove this statement by induction on the sum of the number of edges and vertices of $G$. Assume that $G^{\prime}$ is obtained from $G$ by some Kelmans transformation applied to the vertices $u$ and $v$, where $u$ is the beneficiary and $v$ is the co-beneficiary. Let $w \in N(v) \backslash N(u)$, we can assume the existence of such a vertex, otherwise $G^{\prime}=G$. Let us denote the edge $(v, w) \in E(G)$ by $e=(v, w)$ and the edge $(u, w) \in E\left(G^{\prime}\right)$ by $f=(u, w)$. Then we have

$$
\widehat{c h}(G, \lambda)=\widehat{c h}(G-e, \lambda)+\widehat{c h}(G / e, \lambda)
$$

and

$$
\widehat{c h}\left(G^{\prime}, \lambda\right)=\widehat{c h}\left(G^{\prime}-f, \lambda\right)+\widehat{c h}\left(G^{\prime} / f, \lambda\right) .
$$


Note that $G^{\prime}-f$ can be obtained from $G-e$ by a Kelmans transformation, thus by induction we have

$$
\widehat{c h}(G-e, \lambda) \gg \widehat{c h}\left(G^{\prime}-f, \lambda\right) .
$$

Observe that $G / e$ and $G^{\prime} / f$ are multigraphs, indeed if for some $t \in N_{G}(v)$ the vertex $t$ were adjacent to $w$ than $t w$ became multiple edges in $G / e$. Now we erase all except one copy of all multiple edges to make $G / e$ and $G^{\prime} / f$ simple graphs. (See the remark at the end of the proof.) Let $(G / e)^{*}$ and $\left(G^{\prime} / f\right)^{*}$ be the obtained simple graphs. This way we did not change the chromatic polynomial since the value of $\operatorname{ch}(., \lambda)$ became unchanged for all positive integers, thus the polynomial itself must be unchanged. Another observation is that whenever we erased a multiple edge in $G / e$ we erased a multiple edge in $G^{\prime} / f$ too. On the other hand, for if some $t \in N_{G}(u) \backslash N_{G}(v)$ the vertex $t$ were adjacent to $w$ then it became a multiple edge in $G^{\prime} / f$ while it is a simple edge in $G / e$. Let us erase all edges of the form $\left\{(t, w) \mid t \in N_{G}(u) \backslash N_{G}(w)\right\}$ from the graph $(G / e)^{*}$; let $(G / e)^{* *}$ be the obtained graph. According to Lemma 6.5 we have

$$
\widehat{c h}\left((G / e)^{*}, \lambda\right) \gg \widehat{c h}\left((G / e)^{* *}, \lambda\right) .
$$

Now our last observation is that $\left(G^{\prime} / f\right)^{*}$ can be obtained from $(G / e)^{* *}$ by some Kelmans transformation where $w$ is the beneficiary and $u$ is the co-beneficiary (in $\left(G^{\prime} / f\right)^{*}$ the vertex $u \in V\left((G / e)^{* *}\right)$ became $\left.v \in V\left((G / f)^{*}\right)\right)$. Hence by the induction hypothesis we have

$$
\widehat{c h}\left((G / e)^{* *}, \lambda\right) \gg \widehat{c h}\left(\left(G^{\prime} / f\right)^{*}, \lambda\right) .
$$

Altogether we have

$$
\begin{gathered}
\widehat{c h}(G, \lambda)=\widehat{c h}(G-e, \lambda)+\widehat{c h}(G / e, \lambda)=\widehat{c h}(G-e, \lambda)+\widehat{c h}\left((G / e)^{*}, \lambda\right) \gg \\
\gg \widehat{c h}(G-e, \lambda)+\widehat{c h}\left((G / e)^{* *}, \lambda\right) \gg \widehat{c h}\left(G^{\prime}-f, \lambda\right)+\widehat{c h}\left(\left(G^{\prime} / f\right)^{*}, \lambda\right)= \\
=\widehat{c h}\left(G^{\prime}-f, \lambda\right)+\widehat{c h}\left(G^{\prime} / f, \lambda\right)=\widehat{c h}\left(G^{\prime}, \lambda\right) .
\end{gathered}
$$

By comparing the two ends of the chain of inequalities we obtained the desired result.

Remark 6.6. We avoided the use of multigraphs because we have not defined the Kelmans transformation for multigraphs, although this can be done, see e.g. [3]. In some cases it would have been more convenient to use multigraphs, but in some other cases it would have led to more discussion. Since we were primarily interested in simple graphs we chose the way described in the proof.

\section{Exponential-type graph polynomials}

We call a graph polynomial $f(G, x)$ exponential-type if it satisfies the following identity:

$$
\sum_{\substack{S_{1} \cup S_{2}=V(G), S_{1} \cap S_{2}=\emptyset}} f\left(S_{1}, x\right) f\left(S_{2}, y\right)=f(G, x+y),
$$

where $f(S, x)=f\left(\left.G\right|_{S}, x\right)$. 
Gus Wiseman [20] call these graph polynomials binomial-type.

This is a very special class of graph polynomials, still it has some notable elements: chromatic polynomial, Laplacian polynomial and the following modified matching polynomial: $\bar{M}(G, x)=\sum_{k=0}^{n} m_{k}(G) x^{n-k}$.

The main structure result for exponential-type graph polynomials is the following. For any exponential-type graph polynomial there exists a function $b$ from the isomorphism classes of graphs to the complex numbers such that if

$$
f(G, x)=\sum_{k=1}^{n} a_{k}(G) x^{k}
$$

then

$$
a_{k}(G)=\sum_{\left\{S_{1}, S_{2}, \ldots, S_{k}\right\} \in \mathcal{P}_{k}} b\left(\left.G\right|_{S_{1}}\right) b\left(\left.G\right|_{S_{2}}\right) \ldots b\left(\left.G\right|_{S_{k}}\right)
$$

where the summation goes over the set $\mathcal{P}_{k}$ of the partitions of the vertex set into exactly $k$ sets. We denote this connection by $f(G, x)=f_{b}(G, x)$. It is easy to prove this structure result, but we will not do it. Instead, we use this result as a definition. We can do it since we will not use the original definition.

We can obtain an easy consequence of this structure theorem.

Lemma 7.1. Assume that $b(G) \geq 0$ for all graphs $G$ and

$$
f_{b}(G, x)=\sum_{k=1}^{n} a_{k}(G) x^{k}
$$

Let $H_{1}$ and $H_{2}$ be two graphs on the same vertex set $V$ and let $u, v \in V$. Assume that the following two conditions hold:

- if $u, v \in S$ or $u, v \notin S$ at the same time we have $b\left(\left.H_{1}\right|_{S}\right) \geq b\left(\left.H_{2}\right|_{S}\right)$,

- (cut condition) for all $S$ for which $u, v \in S$ we have

$$
\sum_{\substack{S_{1} \cap S_{2}=\emptyset, S_{1} \cup S_{2}=S \\ u \in S_{1}, v \in S_{2}}} b\left(\left.H_{1}\right|_{S_{1}}\right) b\left(\left.H_{1}\right|_{S_{2}}\right) \geq \sum_{\substack{S_{1} \cap S_{2}=\emptyset, S_{1} \cup S_{2}=S \\ u \in S_{1}, v \in S_{2}}} b\left(\left.H_{2}\right|_{S_{1}}\right) b\left(\left.H_{2}\right|_{S_{2}}\right) .
$$

Then we have $a_{k}\left(H_{1}\right) \geq a_{k}\left(H_{2}\right)$ for all $1 \leq k \leq n$.

Proof. Clearly, the first condition implies that

$$
\sum_{\substack{\left\{S_{1}, S_{2}, \ldots, S_{k}\right\} \in \mathcal{P} \\ u, v \in S_{1}}} b\left(\left.H_{1}\right|_{S_{1}}\right) b\left(\left.H_{1}\right|_{S_{2}}\right) \ldots b\left(\left.H_{1}\right|_{S_{k}}\right) \geq \sum_{\substack{\left\{S_{1}, S_{2}, \ldots, S_{k}\right\} \in \mathcal{P} \\ u, v \in S_{1}}} b\left(\left.H_{2}\right|_{S_{1}}\right) b\left(\left.H_{2}\right|_{S_{2}}\right) \ldots b\left(\left.H_{2}\right|_{S_{k}}\right) .
$$


Similarly, the first and the second condition together imply

$$
\begin{aligned}
\sum_{\substack{\left\{S_{1}, S_{2}, \ldots, S_{k}\right\} \in \mathcal{P} \\
u \in S_{1}, v \in S_{2}}} b\left(\left.H_{1}\right|_{S_{1}}\right) b\left(\left.H_{1}\right|_{S_{2}}\right) \ldots b\left(\left.H_{1}\right|_{S_{k}}\right) \\
=\sum_{\left\{S_{3}, \ldots S_{k}\right\}} b\left(\left.H_{1}\right|_{S_{3}}\right) \ldots b\left(\left.H_{1}\right|_{S_{k}}\right) \sum_{\substack{S_{1} \cup S_{2}=S \\
u \in S_{1}, v \in S_{2}}} b\left(\left.H_{1}\right|_{S_{1}}\right) b\left(\left.H_{1}\right|_{S_{2}}\right) \\
\geq \sum_{\left\{S_{3}, \ldots S_{k}\right\}} b\left(\left.H_{2}\right|_{S_{3}}\right) \ldots b\left(\left.H_{2}\right|_{S_{k}}\right) \sum_{\substack{S_{1} \cup S_{2}=S \\
u \in S_{1}, v \in S_{2}}} b\left(\left.H_{2}\right|_{S_{1}}\right) b\left(\left.H_{2}\right|_{S_{2}}\right) \\
=\sum_{\substack{\left\{S_{1}, S_{2}, \ldots, S_{k}\right\} \in \mathcal{P} \\
u \in S_{1}, v \in S_{2}}} b\left(\left.H_{2}\right|_{S_{1}}\right) b\left(\left.H_{2}\right|_{S_{2}}\right) \ldots b\left(\left.H_{2}\right|_{S_{k}}\right) .
\end{aligned}
$$

By adding up the two equations we obtain

$$
\begin{aligned}
a_{k}\left(H_{1}\right) & =\sum_{\left\{S_{1}, S_{2}, \ldots, S_{k}\right\} \in \mathcal{P}} b\left(\left.H_{1}\right|_{S_{1}}\right) b\left(\left.H_{1}\right|_{S_{2}}\right) \ldots b\left(\left.H_{1}\right|_{S_{k}}\right) \\
& \geq \sum_{\left\{S_{1}, S_{2}, \ldots, S_{k}\right\} \in \mathcal{P}} b\left(\left.H_{2}\right|_{S_{1}}\right) b\left(\left.H_{2}\right|_{S_{2}}\right) \ldots b\left(\left.H_{2}\right|_{S_{k}}\right)=a_{k}\left(H_{2}\right) .
\end{aligned}
$$

Remark 7.2. Naturally, we will use Lemma 7.1 for a graph $G$ and $G^{\prime}$ obtained by Kelmans transformation and $u, v$ beneficiary and co-beneficiary vertices. The first condition is equivalent with the fact that the Kelmans transformation increase (or decrease) the parameter $b($.$) ; indeed, if u, v \in S$ then $\left.G^{\prime}\right|_{S}$ can be obtained from $\left.G\right|_{S}$ by the Kelmans transformation applied to $u$ and $v$. If $u, v \notin S$ then simply $\left.G^{\prime}\right|_{S}=\left.G\right|_{S}$.

One expects that it is easy (or at least not hard) to check the first condition and considerably much harder to check the cut condition. Surprisingly, there are some cases when it is easier to check the cut condition. For instance, let $b(G)=\tau(G)$ be the number of spanning trees. Then

$$
r(G, u, v)=\sum_{\substack{S_{1} \cap S_{2}=\emptyset, S_{1} \cup S_{2}=V(G) \\ u \in S_{1}, v \in S_{2}}} b\left(\left.G\right|_{S_{1}}\right) b\left(\left.G\right|_{S_{2}}\right)
$$

can be interpreted as follows. Let us put an edge $e$ between $u$ and $v$ then $r(G, u, v)$ is exactly the number of spanning trees containing the edge $e$. But this is $\tau(G / e)$. Since $G / e$ and $G^{\prime} / e$ are isomorphic multigraphs we have $r(G, u, v)=r\left(G^{\prime}, u, v\right)$.

We also could have proved the corresponding statement for the coefficients of the (modified) matching polynomial. Since $b(G)=0$ there, except for $G=K_{1}, K_{2}$ we have $b\left(K_{1}\right)=b\left(K_{2}\right)=1$; thus we have to check the first and second conditions for graphs on at most 2 and $4(!)$ vertices, respectively. 


\section{Laplacian polynomial of a graph}

Recall that the Laplacian matrix $L(G)$ of the graph $G$ is $D-A$, where $D$ is the diagonal matrix consisting of the vertex degrees and $A$ is the adjacency matrix. We call the polynomial $L(G, x)=\operatorname{det}(x I-L(G))$ the Laplacian polynomial of the graph $G$, i.e., it is the characteristic polynomial of the Laplacian matrix of $G$. We will write $L(G, x)$ in the form

$$
L(G, x)=\sum_{k=1}^{n}(-1)^{n-k} a_{k}(G) x^{k}
$$

where $a_{k}(G) \geq 0$.

The main result of this section is the following.

Theorem 8.1. The Kelmans transformation decreases the coefficients of the Laplacian polynomial in absolute value, i.e., assume that $G^{\prime}$ is a graph obtained from $G$ by some Kelmans transformation, then

$$
L(G, x) \gg L\left(G^{\prime}, x\right) .
$$

In other words, $a_{k}(G) \geq a_{k}\left(G^{\prime}\right)$ for $k=1, \ldots, n-1$.

To prove this theorem we will prove that the Laplacian polynomial is exponential-type.

Theorem 8.2. The Laplacian polynomial $L(., x)$ is exponential-type with

$$
b(G)=(-1)^{|V(G)|-1} \bar{\tau}(G)=(-1)^{|V(G)|-1}|V(G)| \tau(G) .
$$

We will deduce Theorem 8.2 from the following lemma, which is only a reformulation of Theorem 8.2, but it has the advantage that it appears in the literature explicitly.

Lemma 8.3. [1] Let $\mathcal{F}_{k}(G)$ denote the set of spanning forests of the graph $G$ which have exactly $k$ components. For $F=T_{1} \cup \cdots \cup T_{k} \in \mathcal{F}_{k}$ let $\gamma(F)=\prod_{i=1}^{k}\left|T_{i}\right|$, where $T_{i}$ 's are the connected components of the forest $F$. Then

$$
a_{k}=\sum_{F \in \mathcal{F}_{k}} \gamma(F)
$$

Proof of Theorem 8.2. We can decompose the sum in Lemma 8.3 such that we consider those forests of $\mathcal{F}_{k}$ whose components span the sets $S_{1}, \ldots, S_{k}$. For such a forest $\gamma(F)=$ $\left|S_{1}\right|\left|S_{2}\right| \ldots\left|S_{k}\right|$. The number of such forests is clearly $\tau\left(S_{1}\right) \tau\left(S_{2}\right) \ldots \tau\left(S_{k}\right)$. Altogether we have

$$
a_{k}=\sum_{F \in \mathcal{F}_{k}} \gamma(F)=\sum_{\left\{S_{1}, S_{2}, \ldots, S_{k}\right\}} \bar{\tau}\left(S_{1}\right) \bar{\tau}\left(S_{2}\right) \ldots \bar{\tau}\left(S_{k}\right) \text {. }
$$

Remark 8.4. Hence $(-1)^{n} L(G,-x)=f_{\bar{\tau}}(G, x)$, where $\bar{\tau}(G)=|V(G)| \tau(G)$. So we can use Lemma 7.1 to $f_{\bar{\tau}}(G, x)$. We have to check the two conditions, the first one is the result of Satyanarayana, Schoppmann and Suffel quoted in the introduction of this chapter. 
Theorem 8.5. [19] The Kelmans transformation decreases the number of spanning trees, i.e., assume that $G^{\prime}$ is a graph obtained from $G$ by some Kelmans transformation, then

$$
\tau(G) \geq \tau\left(G^{\prime}\right)
$$

Proof. Let $u$ and $v$ be the beneficiary and the co-beneficiary of the Kelmans transformation, respectively.

Let $R$ be a subset of the edge set $\left\{(u, w) \in E(G) \mid w \in N_{G}(u) \cap N_{G}(v)\right\}$. Let

$$
\mathcal{T}_{R}(G)=\{T \mid T \text { is a spanning tree, } R \subset E(T)\} .
$$

Let $\tau_{R}(G)=\left|\mathcal{T}_{R}(G)\right|$. We will show that for any $R \subseteq\{(u, w) \in E(G) \mid w \in N(u) \cap N(v)\}$, we have $\tau_{R}(G) \geq \tau_{R}\left(G^{\prime}\right)$. For $R=\emptyset$ we immediately obtain the statement of the theorem.

We prove this statement by induction on the lexicographic order of

$$
\left(e(G),\left|N_{G}(u) \cap N_{G}(v)\right|-|R|\right) .
$$

For the empty graph on $n$ vertices the statement is trivial. Thus we assume that we already know that the Kelmans transformation decreases $\tau_{R}\left(G_{1}\right)$ if $e\left(G_{1}\right)<e(G)$ or $e\left(G_{1}\right)=e(G)$, but $\left|N_{G}\left(u_{1}\right) \cap N_{G}\left(v_{1}\right)\right|-\left|R_{1}\right|<\left|N_{G}(u) \cap N_{G}(v)\right|-|R|$.

Now assume that $\left|N_{G}(u) \cap N_{G}(v)\right|-|R|=0$, in other words $R=\{(u, w) \in E(G) \mid w \in$ $N(u) \cap N(v)\}$. Observe that $N_{G^{\prime}}(v)=N_{G}(u) \cap N_{G}(v)$, but since $R \subset E\left(T^{\prime}\right)$ the vertex $v$ must be a leaf in $T^{\prime}$ for any spanning tree $T^{\prime} \in \mathcal{T}_{R}\left(G^{\prime}\right)$.

Now let us consider the following map. Take a spanning tree $T^{\prime}$ which contains the elements of the set $R$. Let us erase the edges between $u$ and $\left(N_{G}(v) \backslash N_{G}(u)\right) \cap N_{T^{\prime}}(u)$ (maybe there is no such edge in the tree) and add the edges between $v$ and $\left(N_{G}(v) \backslash N_{G}(u)\right) \cap N_{T^{\prime}}(u)$. The tree, obtained this way, is an element of $\mathcal{T}_{R}(G)$. This map is obviously injective; if we get an image $T \in \mathcal{T}_{R}(G)$ we simply erase the edges between $v$ and $\left(N_{G}(v) \backslash N_{G}(u)\right) \cap N_{T}(v)$ and add the edges between $u$ and $\left(N_{G}(v) \backslash N_{G}(u)\right) \cap N_{T}(v)$. Hence $\tau_{R}\left(G^{\prime}\right) \leq \tau_{R}(G)$.

Now assume that $|R|<\left|N_{G}(u) \cap N_{G}(v)\right|$. Let $h=(u, w)$ be an edge not in $R$ for which $w \in N_{G}(u) \cap N_{G}(v)$. Then we can decompose $\tau_{R}(G)$ according to $h \in E(T)$ or not. Hence

$$
\tau_{R}(G)=\tau_{R \cup\{h\}}(G)+\tau_{R}(G-h) .
$$

Similarly,

$$
\tau_{R}\left(G^{\prime}\right)=\tau_{R \cup\{h\}}\left(G^{\prime}\right)+\tau_{R}\left(G^{\prime}-h\right) .
$$

Note that $G^{\prime}-h$ can be obtained from $G-h$ by a Kelmans transformation applied to the vertices $u$ and $v$. Since it has fewer edges than $G$ we have

$$
\tau_{R}(G-h) \geq \tau_{R}\left(G^{\prime}-h\right) .
$$

Similarly, $\left|N_{G}(u) \cap N_{G}(v)\right|-|R \cup\{h\}|<\left|N_{G}(u) \cap N_{G}(v)\right|-|R|$, so we have by induction that

$$
\tau_{R \cup\{h\}}(G) \geq \tau_{R \cup\{h\}}\left(G^{\prime}\right) .
$$


Hence

$$
\tau_{R}(G) \geq \tau_{R}\left(G^{\prime}\right)
$$

In particular,

$$
\tau(G)=\tau_{\emptyset}(G) \geq \tau_{\emptyset}\left(G^{\prime}\right)=\tau\left(G^{\prime}\right) .
$$

Now we prove that the function $\bar{\tau}$ satisfies the second condition of Lemma 7.1. The proof of it will be very similar to the previous one.

Theorem 8.6. Let $\bar{\tau}(G)=|V(G)| \tau(G)$, where $\tau(G)$ denotes the number of spanning trees of the graph $G$. Let $G$ be a graph and let $G^{\prime}$ be the graph obtained from $G$ by a Kelmans transformation applied to the vertices $u$ and $v$. Then for all $S$ for which $u, v \in S$ we have

$$
\sum_{\substack{S_{1} \cap S_{2}=\emptyset, S_{1} \cup S_{2}=S \\ u \in S_{1}, v \in S_{2}}} \bar{\tau}\left(\left.G\right|_{S_{1}}\right) \bar{\tau}\left(\left.G\right|_{S_{2}}\right) \geq \sum_{\substack{S_{1} \cap S_{2}=\emptyset, S_{1} \cup S_{2}=S \\ u \in S_{1}, v \in S_{2}}} \bar{\tau}\left(\left.G^{\prime}\right|_{S_{1}}\right) \bar{\tau}\left(\left.G^{\prime}\right|_{S_{2}}\right) .
$$

Proof. We can assume that $S=V(G)$. Let $R$ be a subset of the edge set $\{(u, w) \in$ $E(G) \mid w \in N(u) \cap N(v)\}$. Let

$$
\begin{gathered}
\mathcal{S}(G)_{R}=\left\{\left(T_{1}, T_{2}\right) \mid T_{1}, T_{2} \text { trees, } u \in V\left(T_{1}\right), v \in V\left(T_{2}\right),\right. \\
\left.V\left(T_{1}\right) \cap V\left(T_{2}\right)=\emptyset, V\left(T_{1}\right) \cup V\left(T_{2}\right)=V(G), \quad R \subseteq E\left(T_{1}\right)\right\} .
\end{gathered}
$$

Note that

$$
s(G, u, v):=\sum_{\substack{S_{1} \cap S_{2}=\emptyset, S_{1} \cup S_{2}=S \\ u \in S_{1}, v \in S_{2}}} \bar{\tau}\left(\left.G\right|_{S_{1}}\right) \bar{\tau}\left(\left.G\right|_{S_{2}}\right)=\sum_{\left(T_{1}, T_{2}\right) \in \mathcal{S}(G)_{\emptyset}}\left|V\left(T_{1}\right)\right|\left|V\left(T_{2}\right)\right| .
$$

In general, we introduce the expression

$$
s(G, R, u, v)=\sum_{\left(T_{1}, T_{2}\right) \in \mathcal{S}(G)_{R}}\left|V\left(T_{1}\right)\right|\left|V\left(T_{2}\right)\right| .
$$

We will show that for any $R \subseteq\{(u, w) \in E(G) \mid w \in N(u) \cap N(v)\}$ we have

$$
s(G, R, u, v) \geq s\left(G^{\prime}, R, u, v\right) .
$$

We prove this statement by induction on the lexicographic order of

$$
(|E(G)|,|N(u) \cap N(v)|-|R|) .
$$

For the empty graph on $n$ vertices the statement is trivial. Thus we assume that we already know that the Kelmans transformation decreases $s\left(G_{1}, R_{1}, u_{1}, v_{1}\right)$ if $e\left(G_{1}\right)<e(G)$ or $e\left(G_{1}\right)=e(G)$, but $\left|N\left(u_{1}\right) \cap N\left(v_{1}\right)\right|-\left|R_{1}\right|<|N(u) \cap N(v)|-|R|$. 
Now assume that $|N(u) \cap N(v)|-|R|=0$, in other words, $R=\{(u, w) \in E(G) \mid w \in$ $N(u) \cap N(v)\}$. We prove that $s(G, R, u, v) \geq s\left(G^{\prime}, R, u, v\right)$. Observe that $N_{G^{\prime}}(v)=$ $N(u) \cap N(v)$, but since $R \subseteq T_{1}$ the set $N_{G^{\prime}}(v) \subseteq V\left(T_{1}\right)$. Hence $V\left(T_{2}\right)=\{v\}$. So

$$
s\left(G^{\prime}, R, u, v\right)=(n-1) \tau_{R}\left(G^{\prime}-v\right),
$$

where $\tau_{R}\left(G^{\prime}-v\right)$ denotes the number of spanning trees of $G^{\prime}-v$ which contains the elements of the set $R$. Now let us consider the following map. Take a spanning tree $T^{\prime}$ of $G^{\prime}-v$ which contains the elements of the set $R$, let us erase the edges between $u$ and $\left(N_{G}(v) \backslash N_{G}(u)\right) \cap N_{T^{\prime}}(u)$ (maybe there is no such edge in the tree) and add the edges between $v$ and $\left(N_{G}(v) \backslash N_{G}(u)\right) \cap N_{T^{\prime}}(u)$. The pair of trees, obtained this way, is an element of $\mathcal{S}(G)_{R}$. This map is obviously injective; if we get an image $\left(T_{1}, T_{2}\right) \in \mathcal{S}(G)_{R}$ we simply erase the edges between $v$ and $N_{T_{2}}(v)$ and add the edges between $u$ and $N_{T_{2}}(v)$. Since $n-1 \leq k(n-k)$ for any $1 \leq k \leq n-1$ we have

$$
s\left(G^{\prime}, R, u, v\right)=\sum_{\left(T_{1}, T_{2}\right) \in \mathcal{S}\left(G^{\prime}\right)_{R}} 1 \cdot(n-1) \leq \sum_{\left(T_{1}, T_{2}\right) \in \mathcal{S}(G)_{R}}\left|V\left(T_{1}\right)\right|\left|V\left(T_{2}\right)\right|=s(G, R, u, v) .
$$

Now assume that $|R|<\left|N_{G}(u) \cap N_{G}(v)\right|$. Let $h=(u, w)$ be an edge not in $R$ for which $w \in N_{G}(u) \cap N_{G}(v)$. Then we can decompose $s(G, R, u, v)$ according to $h \in T_{1}$ where $\left(T_{1}, T_{2}\right) \in \mathcal{S}(G)_{R}$ or not. Hence

$$
s(G, R, u, v)=s(G, R \cup\{h\}, u, v)+s(G-h, R, u, v) .
$$

Similarly,

$$
s\left(G^{\prime}, R, u, v\right)=s\left(G^{\prime}, R \cup\{h\}, u, v\right)+s\left(G^{\prime}-h, R, u, v\right) .
$$

Note that $G^{\prime}-h$ can be obtained from $G-h$ by a Kelmans transformation applied to the vertices $u$ and $v$. Since it has fewer edges than $G$ we have

$$
s(G-h, R, u, v) \geq s\left(G^{\prime}-h, R, u, v\right) .
$$

Similarly, $\left|N_{G}(u) \cap N_{G}(v)\right|-|R \cup\{h\}|<\left|N_{G}(u) \cap N_{G}(v)\right|-|R|$, so we have by induction that

$$
s(G, R \cup\{h\}, u, v) \geq s\left(G^{\prime}, R \cup\{h\}, u, v\right) .
$$

Hence

$$
s(G, R, u, v) \geq s\left(G^{\prime}, R, u, v\right) .
$$

In particular,

$$
\begin{aligned}
\sum_{\substack{S_{1} \cap S_{2}=\emptyset, S_{1} \cup S_{2}=S \\
u \in S_{1}, v \in S_{2}}} \bar{\tau}\left(\left.G\right|_{S_{1}}\right) \bar{\tau}\left(\left.G\right|_{S_{2}}\right) & =s(G, \emptyset, u, v) \\
& \geq s\left(G^{\prime}, \emptyset, u, v\right)=\sum_{\substack{S_{1} \cap S_{2}=\emptyset, S_{1} \cup S_{2}=S \\
u \in S_{1}, v \in S_{2}}} \bar{\tau}\left(\left.G^{\prime}\right|_{S_{1}}\right) \bar{\tau}\left(\left.G^{\prime}\right|_{S_{2}}\right) .
\end{aligned}
$$


Proof of Theorem 8.1. Since the Laplace graph is of exponential-type it is enough to check the conditions of Lemma 7.1 for the polynomial $(-1)^{n} L(G,-x)$. This satisfies that $b_{L}(G)=\bar{\tau}(G)=|V(G)| \tau(G) \geq 0$.

If $u, v \in S$, then according Theorem 8.5, $\tau\left(\left.G^{\prime}\right|_{S}\right) \leq \tau\left(\left.G\right|_{S}\right)$ and so $\bar{\tau}\left(\left.G^{\prime}\right|_{S}\right) \leq \bar{\tau}\left(\left.G\right|_{S}\right)$. If $u, v \notin S$ then $\left.G^{\prime}\right|_{S}=\left.G\right|_{S}$ and simply $\bar{\tau}\left(\left.G^{\prime}\right|_{S}\right)=\bar{\tau}\left(\left.G\right|_{S}\right)$.

On the other hand, by Theorem 8.6 we have

$$
\sum_{\substack{S_{1} \cap S_{2}=\emptyset, S_{1} \cup S_{2}=S \\ u \in S_{1}, v \in S_{2}}} \bar{\tau}\left(\left.G\right|_{S_{1}}\right) \bar{\tau}\left(\left.G\right|_{S_{2}}\right) \geq \sum_{\substack{S_{1} \cap S_{2}=\emptyset, S_{1} \cup S_{2}=S \\ u \in S_{1}, v \in S_{2}}} \bar{\tau}\left(\left.G^{\prime}\right|_{S_{1}}\right) \bar{\tau}\left(\left.G^{\prime}\right|_{S_{2}}\right) .
$$

Hence every condition of Lemma 7.1 are satisfied. Thus $a_{k}\left(G^{\prime}\right) \leq a_{k}(G)$ for any $1 \leq k \leq$ $n$.

\section{$9 \quad$ Number of closed walks}

Definition 9.1. The NA-Kelmans transformation is the Kelmans transformation applied to non-adjacent vertices.

Theorem 9.2. The NA-Kelmans transformation increases the number of closed walks of length $k$ for every $k \geq 1$. In other words, $W_{k}\left(G^{\prime}\right) \geq W_{k}(G)$ for $k \geq 1$.

Proof. Let $G$ be an arbitrary graph. Let $G^{\prime}$ be the graph obtained from $G$ by a Kelmans transformation applied to $u$ and $v$, where $u$ is the beneficiary. Let $D(x, y, k)$ denote the number of walks from $x$ to $y$ of length $k$ in $G$. Similarly $R(x, y, k)$ denotes the number of walks from $x$ to $y$ of length $k$ in $G^{\prime}$. If $x, y \neq v$ then for all $k$ we have $R(x, y, k) \geq D(x, y, k)$. Indeed, if we have a walk from $x$ to $y$ of length $k$ we can exchange those $v$ 's to $u$ 's in the walk whose any of the neighbor in the walk is a vertex belonging to $N_{G}(v) \backslash N_{G}(u)$. (It is one of the steps where we use that $u$ and $v$ are not adjacent.) This will give an injective mapping from the walks of $G$ to the set of walks of $G^{\prime}$. (It is not surjective since $\ldots v_{1} u v_{2} \ldots$ never appears in these "image" walks if $v_{1} \in N_{G}(v) \backslash N_{G}(u)$ and $v_{2} \in N_{G}(u) \backslash N_{G}(v)$.) In particular, if $x \neq u, v$ then $R(x, x, k) \geq D(x, x, k)$. On the other hand,

$$
\begin{gathered}
D(u, u, k)+D(v, v, k)=\sum_{x, y \in N_{G}(u)} D(x, y, k-2)+\sum_{x^{\prime}, y^{\prime} \in N_{G}(v)} D\left(x^{\prime}, y^{\prime}, k-2\right) \\
\leq \sum_{x, y \in N_{G}(u)} R(x, y, k-2)+\sum_{x^{\prime}, y^{\prime} \in N_{G}(v)} R\left(x^{\prime}, y^{\prime}, k-2\right) \\
\leq \sum_{x, y \in N_{G^{\prime}}(u)} R(x, y, k-2)+\sum_{x^{\prime}, y^{\prime} \in N_{G^{\prime}}(v)} R\left(x^{\prime}, y^{\prime}, k-2\right)=R(u, u, k)+R(v, v, k) .
\end{gathered}
$$

Hence

$$
W_{k}(G)=\sum_{x \in V(G)} D(x, x, k) \leq \sum_{x \in V(G)} R(x, x, k)=W_{k}\left(G^{\prime}\right) .
$$


Remark 9.3. The statement is not true for any Kelmans transformation. Let $G$ be the 4cycle, and let $u, v$ be two adjacent vertices of $G$. Let us apply the Kelmans transformation to $u$ and $v$. Then $G$ has 32 closed walks of length 4 while $G^{\prime}$ has only 28 closed walks of length 4.

\section{Polynomials of the threshold graphs}

In this section we give some special graph polynomials of the threshold graphs. We start with the Laplacian polynomial (which can be found implicitly in the paper [15] as well, although we give the proof here).

Theorem 10.1. Let $G$ be a threshold graph of Kelmans transformation with degree sequence $d_{1} \geq d_{2} \geq \cdots \geq d_{n}$. Let $t$ be the unique integer for which $d_{t}=t-1$, i.e., for which $v_{1}, \ldots, v_{t}$ induces a clique, but $v_{t}$ and $v_{t+1}$ are not connected. Then the spectra of the Laplacian matrix of $G$ is the multiset

$$
\left\{d_{1}+1, d_{2}+1, \ldots, d_{t-1}+1, d_{t+1}, \ldots, d_{n}, 0\right\} .
$$

In other words, the Laplacian polynomial is

$$
L(G, x)=x \prod_{i=1}^{t-1}\left(x-d_{i}-1\right) \prod_{i=t+1}^{n}\left(x-d_{i}\right) .
$$

Proof. We will use the following well-known facts.

Fact 1. If we add $k$ isolated vertices to the graph $G$ then the Laplacian spectra of the obtained graph consists of the Laplacian spectra of the graph $G$ and $k$ zeros.

Fact 2. ([10]) If the Laplacian spectra of the graph $G$ is $\lambda_{1} \geq \lambda_{2} \geq \cdots \geq \lambda_{n}=0$ then the Laplacian spectra of $\bar{G}$ is $n-\lambda_{1}, n-\lambda_{2}, \ldots, n-\lambda_{n-1}, 0$.

We prove the theorem by induction on the number of vertices of the graph. The claim is trivial for threshold graphs having 1 or 2 vertices. If $v_{1}$ is not adjacent to $v_{n}$ then $v_{n}$ is an isolated vertex and the claim follows from the induction hypothesis and Fact 1 . If $v_{1}$ and $v_{n}$ are adjacent then we observe that $\bar{G}$ has the same structure and $v_{1}$ is isolated vertex in $\bar{G}$. Note that in $\bar{G}$ the vertices $v_{n}, v_{n-1}, \ldots, v_{t+1}, v_{t}$ induce a clique, but $v_{t}$ and $v_{t-1}$ are not adjacent. So we can apply the induction hypothesis to $\bar{G} \backslash\left\{v_{1}\right\}$ obtaining that its Laplacian spectra is $\left\{n-1-d_{n}+1, n-1-d_{n-1}+1, \ldots, n-1-d_{t+1}+1, n-1-d_{t-1}, \ldots, n-1-d_{2}, 0\right\}$. Thus using Fact 2 and $d_{1}=n-1$ we get that the Laplacian spectra of the graph $G$ is $\left\{d_{1}+1, d_{2}+1, \ldots, d_{t-1}+1, d_{t+1}, \ldots, d_{n}, 0\right\}$.

The threshold graphs are also chordal graphs so the roots of their chromatic polynomials are integers. The more precise (and trivial) result is the following. 
Theorem 10.2. Let $G$ be a threshold graph of Kelmans transformation with degree sequence $d_{1} \geq d_{2} \geq \cdots \geq d_{n}$. Let $t$ be the unique integer for which $d_{t}=t-1$, i.e., for which $v_{1}, \ldots, v_{t}$ induce a clique, but $v_{t}$ and $v_{t+1}$ are not connected. Then the chromatic polynomial of the graph $G$ is the following

$$
\operatorname{ch}(G, \lambda)=\prod_{i=1}^{t}(\lambda-i+1) \prod_{i=t+1}^{n}\left(\lambda-d_{i}\right) .
$$

Proof. We can color the clique of size $t$ in $\prod_{i=1}^{t}(\lambda-i+1)$ ways. For $i \geq t+1$, the vertex $v_{i}$ has $d_{i}$ neighbors in the clique induced by $v_{1}, \ldots, v_{t}$, so we can color it in $\lambda-d_{i}$ ways.

It is also easy to determine the independence polynomial of a threshold graph.

Theorem 10.3. Let $G$ be a threshold graph of Kelmans transformation with degree sequence $d_{1} \geq d_{2} \geq \cdots \geq d_{n}$. Let $t$ be the unique integer for which $d_{t}=t-1$, i.e. , for which $v_{1}, \ldots, v_{t}$ induces a clique, but $v_{t}$ and $v_{t+1}$ are not connected. Then the independence polynomial of $G$ is

$$
I(G, x)=(1-x)^{n-t}-x \sum_{i=1}^{t}(1-x)^{n-1-d_{i}} .
$$

Proof. Since every independent set can contain at most one vertex from the clique induced by the vertices of $v_{1}, \ldots, v_{t}$ we can decompose the terms of the independence polynomials as follows. Those independent sets which does not contain any of the vertex $v_{1}, \ldots, v_{t}$ contribute $(1-x)^{n-t}$ to the sum. Those independent sets which contain the vertex $v_{i}$ $(1 \leq i \leq t)$ contribute $-x(1-x)^{n-1-d_{i}}$ to the sum.

Remark 10.4. One can consider the previous theorem as an inclusion-exclusion formula. A more general formula can be found in [7].

It remains to consider the matching polynomials of the threshold graphs. In this case the answer is a bit more complicated. Some notation is in order. First of all, let $M\left(K_{n}, x\right)=H_{n}(x)$ for brevity. Furthermore, let $G$ be a threshold graph with degree sequence $d_{1} \geq d_{2} \geq \cdots \geq d_{n}$. Let $t$ be the unique integer for which $d_{t}=t-1$, i.e., for which $v_{1}, \ldots, v_{t}$ induce a clique, but $v_{t}$ and $v_{t+1}$ are not adjacent and set

$$
M(G, x)=P\left(n, t, d_{t+1}, \ldots, d_{n} ; x\right) .
$$

Then we have

Theorem 10.5.

$$
\begin{gathered}
P\left(n, t, d_{t+1}, \ldots, d_{n} ; x\right)=x P\left(n-1, t, d_{t+1}, \ldots, d_{n-1} ; x\right) \\
-d_{n} P\left(n-1, t-1, d_{t+1}-1, \ldots, d_{n-1}-1 ; x\right)
\end{gathered}
$$


Furthermore,

$$
P\left(n, t, d_{t+1}, \ldots, d_{n} ; x\right)=\sum_{k=0}^{n-t} \widetilde{\sigma}_{k}\left(d_{t+1}, \ldots, d_{n}\right)(-1)^{k} x^{n-t-k} H_{t-k}(x),
$$

where

$$
\tilde{\sigma}_{k}\left(r_{1}, \ldots, r_{m}\right)=\sum_{1 \leq i_{1}<i_{2}<\cdots<i_{k} \leq m}\left(r_{i_{1}}-k+1\right)\left(r_{i_{2}}-k+2\right) \ldots\left(r_{i_{k-1}}-1\right) r_{i_{k}} .
$$

Proof. The recursion follows from the recursion formula for the matching polynomial applied to the edges incident to $v_{n}$ : if $e=\left(v_{i}, v_{n}\right) \in E(G)$ then $G-\left\{v_{i}, v_{n}\right\}$ is a threshold graph with the matching polynomial $P\left(n-1, t-1, d_{t+1}-1, \ldots, d_{n-1}-1 ; x\right)$. If $d_{n}=0$ then the second term vanishes and so it does not cause any problem that $P\left(n-1, t-1, d_{t+1}-\right.$ $\left.1, \ldots, d_{n-1}-1 ; x\right)$ is not the matching polynomial of $G-v_{n}$ and maybe meaningless. The other formula for the matching polynomial easily follows from the recursion formula.

\section{Concluding remarks}

In this last section we wish to make some remarks on the use of the Kelmans transformation. As one can see the threshold graphs of these transformations are very special, so the use of this transformation is restricted to those problems where the extremal graph is conjectured to belong to this class of graphs. But if it is the case then the Kelmans transformation is probably the right tool to attack the problem. One of its main strengths is that it is very simple to work with. The other strength of this transformation is that it is very compatible with the deletion-contraction algorithms; in most of the proofs we used only some special recursion formula for the corresponding polynomial.

Although the Kelmans transformation could handle various problems, the reason why it worked maybe totally different. We try to explain it through two examples. If we are looking for the graph maximizing the spectral radius among graphs with prescribed number of edges then we know from Rowlinson's result [18] that the extremal graph is as "clique-like" as it is possible. The Kelmans transformation works properly because it makes the graphs more "clique-like". Now if we consider the problem of finding the graph maximizing the largest root of the matching polynomial among graphs with prescribed number of edges, the situation is completely different. We believe that the Kelmans transformation works because it generates some large-degree vertices. We conjecture that in this case the extremal graph will be as "star-like" as it is possible: it has as many vertices of degree $n-1$ as it is possible and one more vertex of the clique part of the threshold graph has some additional edges. 


\section{References}

[1] Norman Biggs: Algebraic Graph Theory, Cambridge Tracts in Mathematics, p. 44.

[2] Béla Bollobás: Modern Graph Theory, Springer 2002.

[3] J. Brown, C. Colbourn and J. Devitt: Network transformations and bounding network reliability, Networks 23 (1993), 1-17.

[4] R.A. Brualdi and A.J. Hoffman: On the spectral radius of $(0,1)$ matrices, Linear Alg. Appl. 65 (1985), 133-146.

[5] Péter Csikvári: On a conjecture of V. Nikiforov, Disc. Math. 309, (2009), 4522-4526.

[6] E. J. Cockayne and P. J. Lorimer: The Ramsey number for stripes, J. Austral. Math. Soc. 19 (1975), 252-256.

[7] Jianquo Qian, Andreas Dress and Yan Wang: On the dependence polynomial of a graph, Euro. J. Combin. 28, (2007), 337-346.

[8] David C. Fisher and Jennifer Ryan: Bounds on the largest root of the matching polynomial, Disc. Math. 110, (1992), 275-278.

[9] C. D. Godsil: Algebraic Combinatorics, Chapman and Hall, New York 1993.

[10] C. Godsil and G. Royle: Algebraic Graph Theory, Springer-Verlag, Graduate Texts in Mathematics, 2001, pp. 280 and 282.

[11] C. D. Godsil and I. Gutman: On the theory of the matching polynomial, J. Graph Theory 5 (2006), 137-144.

[12] O. J. Heilmann and E. H. Lieb: Theory of monomer-dimer systems, Commun. Math. Physics 25 (1972), 190-232.

[13] A. K. Kelmans: On graphs with randomly deleted edges, Acta. Math. Acad. Sci. Hung. 37 (1981), 77-88.

[14] Vadim E. Levit and Eugen Mandrescu: The independence polynomial of a graph- a survey, Proc. 1st International Conference on Algebraic Informatics, Thessaloniki, (Ed. S. Bozapalidis, A. Kalampakas and G. Rahonis), Aristotle Univ. 233-254, 2005.

[15] Russel Merris: Degree-maximal graphs are Laplacian integral, Linear Alg. Appl. 199, Supplement 1, 1994, 381-389.

[16] Russel Merris: Threshold graphs, in: Proceedings of the Prague Mathematical Conference, 1996, 205-210.

[17] R. C. Read: An introduction to Chromatic Polynomials, J. Combin. Theory 4 (1968), $52-71$.

[18] Peter Rowlinson: On the maximal index of graphs with a prescribed number of edges, Linear Alg. Appl. 110 (1988), 43-53.

[19] A. Satyanarayana, L. Schoppman and C. L. Suffel: A reliability-improving graph transformation with applications to network reliability, Networks 22 (1992), 209-216.

[20] Gus Wiseman: Set maps, umbral calculus, and the chromatic polynomial., Disc. Math. 308 (2008), 3551-3564. 